\title{
Enhanced dust heating in the bulges of early-type spiral galaxies ${ }^{\star}$
}

\author{
C. W. Engelbracht ${ }^{1}$, L. K. Hunt ${ }^{2}$, R. A. Skibba ${ }^{1}$, J. L. Hinz ${ }^{1}$, D. Calzetti ${ }^{3}$, K. D. Gordon ${ }^{4}$, H. Roussel ${ }^{5}$, A. F. Crocker ${ }^{3}$, \\ K. A. Misselt ${ }^{1}$, A. D. Bolatto ${ }^{6}$, R. C. Kennicutt ${ }^{7}$, P. N. Appleton ${ }^{8}$, L. Armus ${ }^{9}$, P. Beirão ${ }^{9}$, B. R. Brandl ${ }^{10}$, \\ K. V. Croxall ${ }^{11}$, D. A. Dale ${ }^{12}$, B. T. Draine ${ }^{13}$, G. Dumas ${ }^{14}$, A. Gil de Paz ${ }^{15}$, B. Groves ${ }^{10}$, C.-N. Hao ${ }^{16}$, B. D. Johnson ${ }^{7}$, \\ J. Koda ${ }^{17}$, O. Krause ${ }^{14}$, A. K. Leroy ${ }^{18, \star \star}$, S. E. Meidt ${ }^{14}$, E. J. Murphy ${ }^{9}$, N. Rahman ${ }^{19}$, H.-W. Rix ${ }^{14}$, \\ K. M. Sandstrom ${ }^{14}$, M. Sauvage ${ }^{20}$, E. Schinnerer ${ }^{14}$, J.-D. T. Smith ${ }^{11}$, S. Srinivasan ${ }^{5}$, L. Vigroux ${ }^{5}$, F. Walter ${ }^{14}$, \\ B. E. Warren ${ }^{21}$, C. D. Wilson ${ }^{19}$, M. G. Wolfire ${ }^{6}$, and S. Zibetti ${ }^{14}$
}

(Affiliations are available in the online edition)

Received 31 March 2010 / Accepted 23 April 2010

\begin{abstract}
Stellar density and bar strength should affect the temperatures of the cool ( $T \sim 20-30 \mathrm{~K})$ dust component in the inner regions of galaxies, which implies that the ratio of temperatures in the circumnuclear regions to the disk should depend on Hubble type. We investigate the differences between cool dust temperatures in the central $3 \mathrm{kpc}$ and disk of 13 nearby galaxies by fitting models to measurements between 70 and $500 \mu \mathrm{m}$. We attempt to quantify temperature trends in nearby disk galaxies, with archival data from Spitzer/MIPS and new observations with Herschel/SPIRE, which were acquired during the first phases of the Herschel observations for the KINGFISH (Key Insights on Nearby Galaxies: a Far-Infrared Survey with Herschel) sample. We fit single-temperature modified blackbodies to far-infrared and submillimeter measurements of the central and disk regions of galaxies to determine the temperature of the component(s) emitting at those wavelengths. We present the ratio of central-region-todisk-temperatures of the cool dust component of 13 nearby galaxies as a function of morphological type. We find a significant temperature gradient in the cool dust component in all galaxies, with a mean center-to-disk temperature ratio of $1.15 \pm 0.03$. The cool dust temperatures in the central $\sim 3 \mathrm{kpc}$ of nearby galaxies are $23( \pm 3) \%$ hotter for morphological types earlier than Sc, and only $9( \pm 3) \%$ hotter for later types. The temperature ratio is also correlated with bar strength, with only strongly barred galaxies having a ratio over 1.2. The strong radiation field in the high stellar density of a galactic bulge tends to heat the cool dust component to higher temperatures, at least in early-type spirals with relatively large bulges, especially when paired with a strong bar.
\end{abstract}

Key words. galaxies: ISM - infrared: galaxies - submillimeter: galaxies - dust, extinction

\section{Introduction}

The infrared emission from galaxies contains roughly half of the entire energy budget in the Universe (e.g., Hauser \& Dwek 2001). In addition to providing information on the amount of attenuation suffered by the stellar light, the infrared emission provides clues to important physical quantities, such as the metal, dust, and cold gas content of galaxies (e.g., Draine et al. 2007, Bernard et al. 2008). Infrared spectral energy distributions (SEDs), especially those extending into the submillimeter regime, can be used to measure the dust mass and temperature in galaxies (e.g., Dunne et al. 2000, 2001; Seaquist et al. 2004; Vlahakis et al. 2005; Draine et al. 2007; Willmer et al. 2009; Liu et al. 2010).

These temperature measurements have shown that the dust is warmer in the centers of galaxies than in the outskirts (e.g., Alton et al. 1998; Radovich et al. 2001; Melo et al. 2002; Dupac et al. 2003). Cool dust at roughly the same temperature in spiral disks is detected globally at longer wavelengths $(850 \mu \mathrm{m}$ and $1.3 \mathrm{~mm}$; Siebenmorgen et al. 1999; Dunne et al. 2000, 2001; Vlahakis et al. 2005), but there is also some evidence of a

* Herschel is an ESA space observatory with science instruments provided by the European-led Principal Investigator consortia and with important participation from NASA.

$\star \star$ Hubble Fellow. warmer temperature component associated with the central regions. Warmer dust temperatures tend to be associated with significant star-formation activity, the resulting intense interstellar radiation field (Stevens et al. 2005) and the earlier Hubble type (Bendo et al. 2003).

Here we use the infrared SEDs, from 70 to $500 \mu \mathrm{m}$ (a range which should be dominated by emission from grains in thermal equilibrium with the radiation field, e.g., Popescu et al. 2000, Engelbracht et al. 2008), of a local sample of 13 galaxies spanning the range of spiral galaxies in the Hubble sequence, to derive the temperature of the cool $(T \sim 20-30 \mathrm{~K})$ dust component of the central region and the disks separately, and investigate differences in the dust heating in the two regions. To achieve this goal, we use the 250, 350, and $500 \mu \mathrm{m}$ Herschel/SPIRE (Spectral and Photometric Imaging REceiver; Griffin et al. 2010) images of the galaxies combined with the Spitzer/MIPS (Multiband Imaging Photometer for Spitzer; Rieke et al. 2004) 70 and $160 \mu \mathrm{m}$ images. Eventually we will use PACS (Photodetector Array Camera and Spectrometer; Poglitsch et al. 2010) imaging for this study, but at the time of this writing, the data were not yet available. These galaxies have little nuclear activity that might heat the dust, with only NGC 1097 having a strongly active nucleus, so this is the first study to cleanly separate the far-infrared properties of central and disk regions in a sample of normal galaxies. 
Until recently, little work has been done to dissect the dust emission in galaxies into sub-galactic components, owing to the general paucity of infrared images with the required angular resolution and to poor long-wavelength sensitivity. Some recent work includes a study of the galaxy pair NGC 1512/1510 (a target also discussed in this paper), which finds that the dust temperature in the central region of NGC 1512 is slightly higher than in the disk and that there is a significantly higher fraction of warm dust, in agreement with the center of NGC 1512 being a starburst (Liu et al. 2010). Work by Muñoz-Mateos et al. (2009) examines radial trends in dust properties in a number of nearby galaxies.

The Herschel Space Observatory promises to yield a breakthrough in the study of subgalactic components in galaxies. This paper is the first investigation to leverage the longest wavelength Herschel data available for the KINGFISH (key insights on nearby galaxies: a far-infrared survey with Herschel; this program is largely derived from SINGS, the Spitzer infrared nearby galaxy survey by Kennicutt et al. 2003) sample of nearby galaxies, which will eventually total 61. Companion papers from the science demonstration phase for this program showcase the shorter wavelength imaging (Sandstrom et al. 2010) and the spectroscopic data (Beirão et al. 2010).

Here we present new SPIRE images acquired during the first few months of Herschel operations, in the context of the KINGFISH open-time key program. We divide each galaxy into two spatially-resolved zones: the circumnuclear region and the surrounding disk. Then we compare central temperatures with those for the disk.

\section{Observations and data reduction}

We observed 33 nearby galaxies with the SPIRE instrument on Herschel (Pilbratt et al. 2010) in the scan map mode as part of the KINGFISH program over the period of November 2009 to January 2010. They were observed in the scan mode out to 1.5 optical radii, to depths of $(3.2,2.5$, and 2.9$) \mathrm{mJy} /$ beam at (250, 350, and 500) $\mu \mathrm{m}$. They were reduced using the standard calibration products and algorithms available in HIPE (the Herschel interactive processing environment; Ott 2010), version 1.2.5 or 2.0.0 (whichever version was the latest available when the target was observed, since the SPIRE calibration is not sensitive to this range of HIPE versions). We modified the offsetsubtraction algorithm in the pipeline, by requiring it to measure the offset only outside bright objects in the field (usually the galaxy that we targeted) - this procedure reduced a small negative offset (6\% in the first galaxy we observed, NGC 4559) in the background in the same rows and columns as the source to an undetectable level. Otherwise, the data reduction was performed using the default pipeline.

\section{Analysis}

Of the 33 galaxies observed, we selected 13 which had both a large extent (i.e., greater than several 160/500 $\mu \mathrm{m}$ beam diameters, or $\sim 2^{\prime}$ ) and a bright disk (brighter than $\sim 0.5 \mathrm{Jy}$ at $500 \mu \mathrm{m}$, excluding the nucleus). These galaxies are listed in Table 1. For the wavelengths with significantly smaller beams (i.e., 70, 250, and $350 \mu \mathrm{m}$ ) than the largest used here, we convolved the data to the SPIRE $500 \mu \mathrm{m}$ resolution using the kernels described by Gordon et al. (2008) and updated by those authors for Herschel. For each galaxy, we computed masks that isolated the disk and central regions. The outer mask is an ellipse sized to encompass the disk where it contrasts strongly with the background,
Table 1. Galaxy sample and computed temperatures.

\begin{tabular}{rrrrrr}
\hline \hline Name & $\begin{array}{r}\text { Hubble } \\
\text { type }\end{array}$ & $\begin{array}{r}D^{a} \\
(\mathrm{Mpc})\end{array}$ & $\begin{array}{r}\text { Spectral }^{b} \\
\text { type }\end{array}$ & $\begin{array}{r}T_{\text {disk }}{ }^{c} \\
(\mathrm{~K})\end{array}$ & $\begin{array}{r}T_{\text {center }^{c}} \\
(\mathrm{~K})\end{array}$ \\
\hline NGC 0628 & SAc & 7.3 & HII? & $22.7 \pm 0.4$ & $24.2 \pm 0.5$ \\
NGC 0925 & SABd & 9.0 & HII & $22.3 \pm 0.4$ & $23.9 \pm 0.5$ \\
NGC 1097 & SBb & 19.1 & Sy1 & $23.4 \pm 0.5$ & $28.7 \pm 0.7$ \\
NGC 1291 & SB0/a & 10.4 & $\ldots$ & $18.3 \pm 0.3$ & $27.6 \pm 0.6$ \\
NGC 1512 & SBa & 14.4 & HII & $21.3 \pm 0.4$ & $27.3 \pm 0.6$ \\
NGC 3621 & SAd & 6.9 & $\ldots$ & $21.9 \pm 0.4$ & $25.6 \pm 0.6$ \\
NGC 4559 & SABcd & 8.5 & HII & $22.7 \pm 0.4$ & $24.2 \pm 0.5$ \\
NGC 4594 & SAa & 9.4 & Sy1.9 & $21.3 \pm 0.4$ & $22.9 \pm 0.4$ \\
NGC 4631 & SBd & 7.6 & HII & $24.5 \pm 0.5$ & $27.7 \pm 0.6$ \\
NGC 5055 & SAbc & 10.2 & Transition2 & $22.0 \pm 0.4$ & $25.3 \pm 0.5$ \\
NGC 5457 & SABcd & 7.1 & HII & $23.4 \pm 0.5$ & $23.9 \pm 0.5$ \\
NGC 6946 & SABcd & 6.8 & HII & $24.5 \pm 0.5$ & $26.6 \pm 0.6$ \\
NGC 7331 & SAb & 14.9 & Transition2 & $23.2 \pm 0.4$ & $26.2 \pm 0.6$ \\
\hline
\end{tabular}

Notes. ${ }^{(a)}$ As listed in NED: The NASA/IPAC Extragalactic Database (NED) is operated by the Jet Propulsion Laboratory, California Institute of Technology, under contract with the National Aeronautics and Space Administration. ${ }^{(b)}$ From NED or Ho et al. 1997. ${ }^{(c)}$ Includes uncertainty in the photometric calibration.

at $1.2,6.6,2.7,1.4$, and $0.8 \mathrm{MJy} / \mathrm{sr}$ at $70,160,250,350$, and $500 \mu \mathrm{m}$ (where the SPIRE data have been converted to MJy/sr as described below), respectively. The inner mask is a circle centered on the galaxy peak, with a minimum size chosen to be as small as possible (i.e., set by the MIPS $160 \mu \mathrm{m}$ beam) and with a radius inversely proportional to the distance to the galaxy. Thus, a similar physical region of $\sim 3 \mathrm{kpc}$ diameter (in which one would expect to find structures like a bulge, bar, ring, and/or inner disk, e.g., see Erwin \& Sparke 2002; Athanassoula \& Martinet 1980; and Knapen 2005) is sampled in the central region of each galaxy. Our results are not sensitive to a modest change in the aperture size, for example, similar results are achieved with a fixed central aperture size of $40^{\prime \prime}$ for each galaxy. A sample mask is shown in Fig. 1.

We measured flux densities in these apertures for each of the 3 Herschel/SPIRE bands, as well as the Spitzer/MIPS 70 and $160 \mu \mathrm{m}$ bands. The Herschel/SPIRE data were calibrated in units of Jy/beam, which we converted to Jy by assuming beam sizes of 371,720 , and 1543 square arcseconds in the 250,350, and $500 \mu \mathrm{m}$ bands, respectively (Swinyard et al. 2010). We assign uncertainties as the quadratic sum of the data-dependent scatter and (by far the larger term) the uncertainty in the absolute calibration, taken to be $5 \%$ and $12 \%$ for Spitzer/MIPS at 70 and $160 \mu \mathrm{m}$ (Gordon et al. 2007 and Stansberry et al. 2007, respectively) and $16 \%$ (the quadratic sum of the $15 \%$ absolute calibration uncertainty from Swinyard et al. 2010 and a $2 \%$ uncertainty in the size of the beam) for SPIRE data.

To each set of data, we fit a blackbody with a frequencydependent emissivity of 1.5 . Our conclusions do not change if values of 1 or 2 are used, although the temperatures increase or decrease, respectively. The uncertainties in the temperatures were computed via a Monte Carlo simulation, in which we performed 10000 trials where the photometric measurements were allowed to vary in a normal distribution with a standard deviation indicated by the photometric uncertainty. An example of fits and data points is shown in Fig. 2, where we can see that the $250 \mu \mathrm{m}$ flux density is underpredicted by this simple model. This happens frequently in our dataset, and may be a sign that multiple temperature components are present in the regions we measured. The values of the Herschel/SPIRE uncertainty indicated by the 

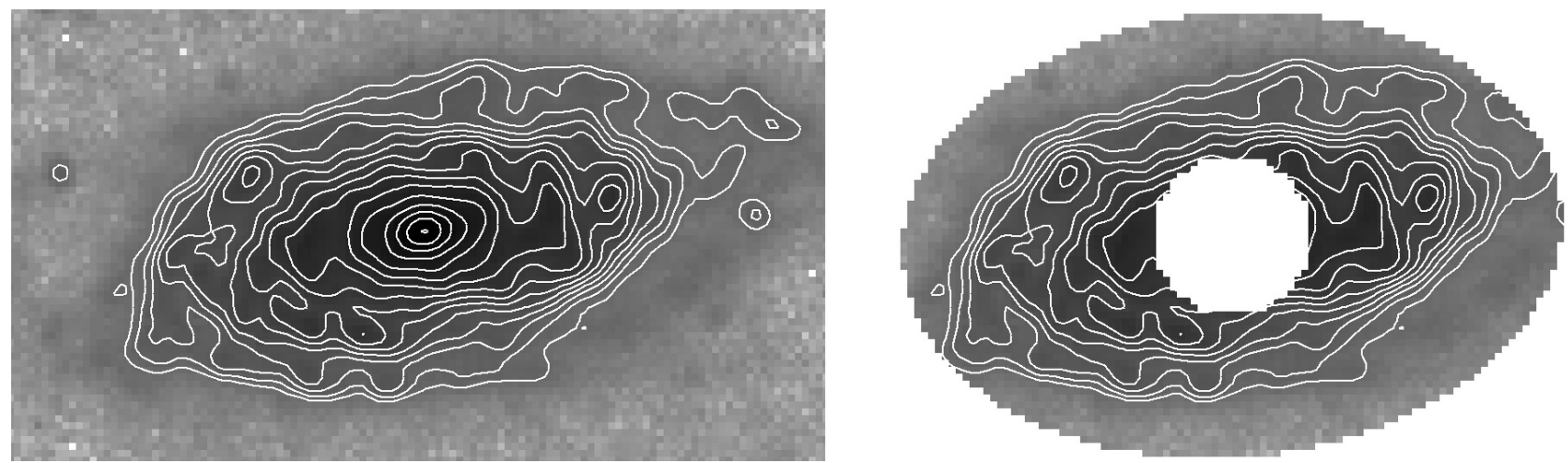

Fig. 1. Example of the masking approach used, shown here at $250 \mu \mathrm{m}$ on a cropped image of NGC 5055. The image on the left shows the full, reduced image, while to the image on the right we have applied a mask (the blank, white regions) to all data but the disk. Both images have been scaled logarithmically, from -0.01 to $5 \mathrm{Jy} / \mathrm{beam}$. The contour levels are scaled logarithmically from 0.1 to $5 \mathrm{Jy} / \mathrm{beam}$. Each panel is $6^{\prime}$ across.

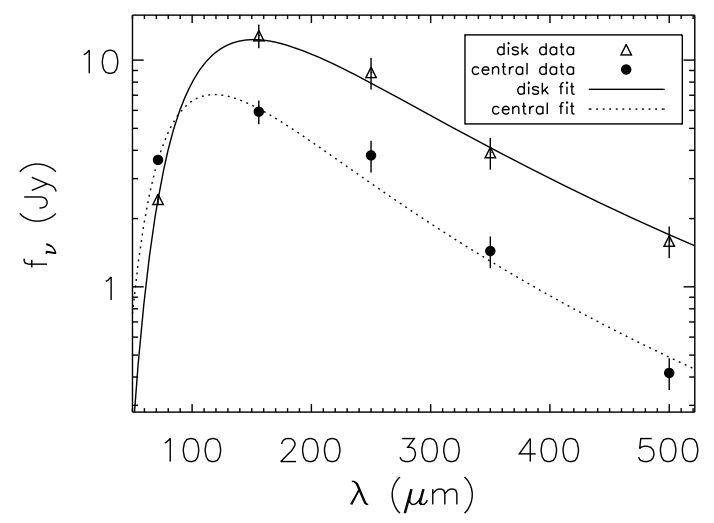

Fig. 2. Far-infrared SEDs of the disk and central region of NGC 1512, each fit by a blackbody function modified by a $\lambda^{-1.5}$ emissivity.

uncertainty in the absolute calibration were allowed to vary together; i.e., the calibration uncertainty was assumed to be correlated among the three bands. In contrast, we allowed the MIPS uncertainties to vary independently; while not being strictly independent (see Stansberry et al. 2007), the $160 \mu \mathrm{m}$ calibration observations, in particular, are dominated by observational scatter and can be treated independently of the $70 \mu \mathrm{m}$ band. The temperature of each component is taken to be the temperature of the best-fit model, with an uncertainty determined by the Monte Carlo simulation.

\section{Results}

For each galaxy, we computed a ratio of the central to disk temperature (from data at wavelengths longer than $70 \mu \mathrm{m}$ ) of the cool dust component. The average ratio of central-to-disk temperatures in this sample is $1.15 \pm 0.03$. We have plotted this ratio against morphological type in Fig. 3, where the average ratio is $1.23 \pm 0.03$ for types earlier than Sc and $1.09 \pm 0.03$ for later types. The trend is poorly described by a line, because it has a correlation coefficient of 0.66 . We plot the ratio against bar strength (as defined by the morphological type) in Fig. 4, where the average ratio is $1.29 \pm 0.04$ for strong bars and $1.09 \pm 0.03$ for weak bars.

A significant $(3 \sigma)$ correlation between temperature ratio and Hubble type is observed in Fig. 3, although the correlation is only marginal, at $2 \sigma$, if the most significant point (NGC 1291) is

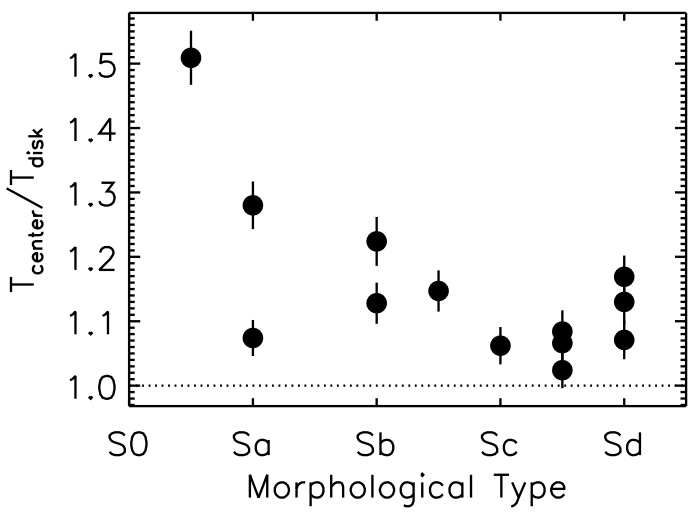

Fig. 3. Ratio of central dust temperature to disk dust temperature as a function of morphological type. The dotted line is drawn at a ratio of 1 (i.e., at equal temperatures) to guide the eye.

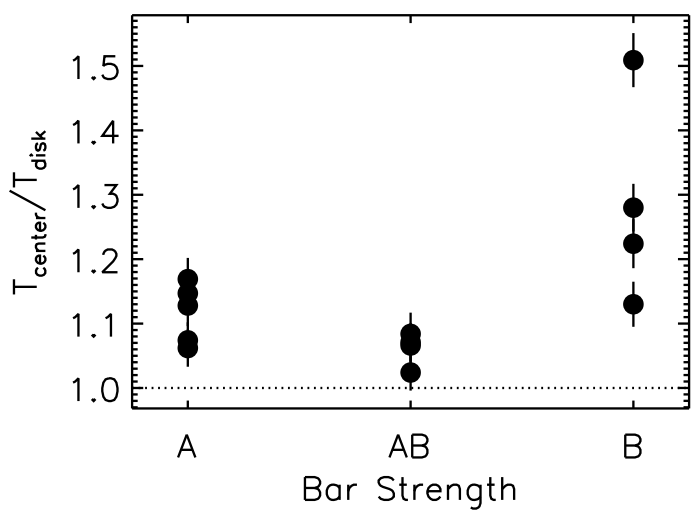

Fig. 4. Ratio of central dust temperature to disk dust temperature as a function of bar strength, as indicated by morphological type. The dotted line is drawn at a ratio of 1 (i.e., at equal temperatures) to guide the eye.

removed from the correlation. This trend suggests that the dominant stellar bulges in early-type spirals are able to heat the cool dust in the ISM to greater temperatures than their late-type counterparts. The effect is most pronounced in the earliest type galaxy in our sample, NGC 1291, in which the dust in the central region is almost $50 \%$ warmer than the dust in the disk. In comparing this to the spectral types in Table 1, we have found no obvious correlation between the temperature ratio and the presence of a central nonthermal source in our galaxies. 
Bars may play an important role in driving the correlation of temperature ratio with morphology. As shown in Fig. 4, a strong bar is correlated with a high center/disk temperature ratio, although the scatter in the strong-bar ratios is large. This could occur through bar-induced starbursts, since mid-infrared colors are redder in barred galaxies, implying warmer dust temperatures (Huang et al. 1996; Roussel et al. 2001). Barred galaxies also tend to have higher star-formation rates (SFRs) than unbarred galaxies, possibly because bars drive gas into the inner regions where it can pile up in the inner dynamical resonances associated with rings (Hawarden et al. 1986). The bar-driven gas transport has been shown in CO observations (e.g., Sakamoto et al. 1999). Stars will form quite readily in such conditions, where the gas transported by the bar fuels star formation (Erwin \& Sparke 2002; Böker et al. 2008; Comerón et al. 2008). In fact, the frequency of inner rings in early-type barred spirals (Hunt \& Malkan 1999; Erwin \& Sparke 2002) could conspire to produce the effect seen in our sample. Similarly, bars are more frequently found in bulge-dominated galaxies (e.g., Masters et al. 2010), which would also contribute to the trends we see. The concentrations of $\mathrm{CO}$ gas in the centers have been observed in early-type spiral galaxies with intense star formation (Koda et al. 2005). In addition to a dependence on stellar density, the higher temperature ratios in the early types shown here could therefore be associated with bar-induced star formation. This is consistent with the result we get if the $70 \mu \mathrm{m}$ fluxes are not included in the modified blackbody fits. Without them, the correlation between temperature ratio and either Hubble type or bar strength disappears; i.e., measurements at wavelengths shorter than the peak emission are required to determine the dust temperature accurately.

\section{Conclusion}

We used far-infrared data from Spitzer and submillimeter data from Herschel to compute separate SEDs for the center and disk regions of 13 nearby galaxies observed as part of the KINGFISH program. We fit those SEDs (at wavelengths longer than $70 \mu \mathrm{m}$ ) with blackbody functions (modified by a frequency-dependent emissivity) to compute temperatures. On average, the cool dust temperature of the central component is $15 \pm 3 \%$ hotter than the disk. We find that the central temperature is higher than the disk by $20 \%$ to $50 \%$ in galaxies of type S0 to Sb, but only $9 \%$ higher in later types. This ratio is also higher (at $1.29 \pm$ 0.04 ) in strongly barred galaxies than in weakly barred galaxies (at $1.09 \pm 0.03$ ).

The data therefore indicate that the large (or "classical") grains that dominate the far-infrared and submillimeter emission are warmer in the centers of those galaxies with a substantial bulge and/or a strong bar. This may simply be caused by the higher density of the radiation field in the centers of early-type spirals, enhanced star formation due to the bar, or some combination of the two. A cleaner separation of morphological components (perhaps with larger samples and/or less distant galaxies) and a more thorough assessment of the density of starlight and star formation activity, plus an evaluation of the impact of central nonthermal sources, may help separate these effects.

The analysis presented here illustrates the power of Herschel observations in characterizating the spatially resolved distribution of dust in nearby galaxies. This power will grow with the use of better-resolved far-infrared SEDs as measured by PACS
(Poglitsch et al. 2010), which will let us measure smaller and/or more distant galaxies and determine radial trends of dust temperature.

Acknowledgements. The following institutes have provided hardware and software elements to the SPIRE project: University of Lethbridge, Canada; NAOC, Beijing, China; CEA Saclay, CEA Grenoble, and OAMP in France; IFSI, Rome, and University of Padua, Italy; IAC, Tenerife, Spain; Stockholm Observatory, Sweden; Cardiff University, Imperial College London, UCL-MSSL, STFCRAL, UK ATC Edinburgh, and the University of Sussex in the UK. Funding for SPIRE has been provided by the national agencies of the participating countries and by internal institute funding: CSA in Canada; NAOC in China; CNES, CNRS, and CEA in France; ASI in Italy; MCINN in Spain; Stockholm Observatory in Sweden; STFC in the UK; and NASA in the USA. Additional funding support for some instrument activities has been provided by ESA. We would also like to thank the anonymous referee whose comments helped improve this paper.

\section{References}

Alton, P. B., Trewhella, M., Davies, J. I., et al. 1998, A\&A, 335, 807 Athanassoula, E., \& Martinet, L. 1980, A\&A, 87, L10

Beirão, P., et al. 2010, A\&A, 518, L60

Bendo, G. J., Joseph, R. D., Wells, M., et al. 2003, AJ, 125, 2361

Bernard, J.-P., Reach, W. T., Paradis, D., et al. 2008, AJ, 136, 919

Böker, T., Falcón-Barroso, J., Schinnerer, E., Knapen, J. H., \& Ryder, S. 2008, AJ, 135, 479

Comerón, S., Knapen, J. H., \& Beckman, J. E. 2008, A\&A, 485, 695 Dale, D. A., \& Helou, G. 2002, ApJ, 576, 159

Dale, D. A., Gil de Paz, A., Gordon, K. D., et al. 2007, ApJ, 655, 863 Dale, D. A., Cohen, S. A., Johnson, L. C., et al. 2009, ApJ, 703, 517 Draine, B. T., \& Li, A. 2007, ApJ, 657, 810

Draine, B. T., Dale, D. A., Bendo, G., et al. 2007, ApJ, 663, 866

Dunne, L., \& Eales, S. A. 2001, MNRAS, 327, 697

Dunne, L., Eales, S., Edmunds, M., et al. 2000, MNRAS, 315, 115

Dupac, X., del Burgo, C., Bernard, J.-P., et al. 2003, MNRAS, 344, 105

Engelbracht, C. W., Rieke, G. H., Gordon, K. D., et al. 2008, ApJ, 678, 804 Erwin, P., \& Sparke, L. S. 2002, AJ, 124, 65

Gordon, K. D., Engelbracht, C. W., Fadda, D., et al. 2007, PASP, 119, 1019

Gordon, K. D., Engelbracht, C. W., Rieke, G. H., et al. 2008, ApJ, 682, 336 Griffin, M. J., et al. 2010, A\&A, 518, L3

Hauser, M. G., \& Dwek, E. 2001, ARA\&A, 39, 249

Hawarden, T. G., Mountain, C. M., Leggett, S. K., \& Puxley, P. J. 1986, MNRAS, $221,41 \mathrm{P}$

Ho, L. C., Filippenko, A. V., \& Sargent, W. L. W., 1997, ApJS, 112, 315

Huang, J. H., Gu, Q. S., Su, H. J., et al. 1996, A\&A, 313, 13

Hunt, L. K., \& Malkan, M. A. 1999, ApJ, 516, 660

Kennicutt, R. C., Jr., Armus, L., Bendo, G., et al. 2003, PASP, 115, 928

Knapen, J. H. 2005, A\&A, 429, 141

Koda, J., Okuda, T., Nakanishi, K., et al. 2005, A\&A, 431, 887

Liu, G., Calzetti, D., Yun, M. S., et al. 2010, AJ, 139, 1190

Masters, K. L., Nichol, R. C., Hoyle, B., et al. 2010, MNRAS, submitted [arXiv: 1003.0449]

Melo, V. P., Pérez García, A. M., Acosta-Pulido, J. A., Muñoz-Tuñón, C., \& Rodríguez Espinosa, J. M. 2002, ApJ, 574, 709

Muñoz-Mateos, J. C., Gil de Paz, A., Boissier, S., et al. 2009, ApJ, 701, 1965 Ott, S. 2010, in Astronomical Data Analysis Software and Systems XIX, ed. Y.

Mizumoto, K.-I. Morita, \& M. Ohishi, ASP Conf. Ser., in press

Pilbratt, G. L., et al. 2010, A\&A, 518, L1

Poglitsch, A., et al. 2010, A\&A, 518, L2

Popescu, C. C., Misiriotis, A., Kylafis, N. D., Tuffs, R. J., \& Fischera, J. 2000, A\&A, 362, 138

Radovich, M., Kahanpää, J., \& Lemke, D. 2001, A\&A, 377, 73

Rieke, G. H., Young, E. T., Engelbracht, C. W., et al. 2004, ApJS, 154, 25

Roussel, H., Sauvage, M., Vigroux, L., et al. 2001, A\&A, 372, 406

Sakamoto, K., Okumura, S. K., Ishizuki, S., \& Scoville, N. Z. 1999, ApJ, 525, 691

Sandstrom, K., et al. 2010, A\&A, 518, L59

Seaquist, E., Yao, L., Dunne, L., \& Cameron, H. 2004, MNRAS, 349, 1428

Siebenmorgen, R., Krügel, E., \& Chini, R. 1999, A\&A, 351, 495

Stansberry, J. A., Gordon, K. D., Bhattacharya, B., et al. 2007, PASP, 119, 1038

Stevens, J. A., Amure, M., \& Gear, W. K. 2005, MNRAS, 357, 361

Swinyard, B. M., Ade, P., Baluteau, J-P., et al. 2010, A\&A, 518, L4

Vlahakis, C., Dunne, L., \& Eales, S. 2005, MNRAS, 364, 1253

Willmer, C. N. A., Rieke, G. H., Le Floc'h, E., et al. 2009, AJ, 138, 146

Page 5 is available in the electronic edition of the journal at http://www . aanda. org 
1 Steward Observatory, Univ. of Arizona, Tucson, AZ 85721, USA e-mail: cengelbracht@as.arizona.edu

2 INAF - Osservatorio Astrofisico di Arcetri, Largo E. Fermi 5, 50125 Firenze, Italy

3 Department of Astronomy, University of Massachusetts, Amherst, MA 01003, USA

${ }^{4}$ Space Telescope Science Institute, 3700 San Martin Drive, Baltimore, MD 21218, USA

5 Institut d'Astrophysique de Paris, UMR7095 CNRS, Université Pierre \& Marie Curie, 98bis boulevard Arago, 75014 Paris, France

${ }^{6}$ Department of Astronomy, University of Maryland, College Park, MD 20742, USA

7 Institute of Astronomy, University of Cambridge, Madingley Road, Cambridge CB3 OHA, UK

8 NASA Herschel Science Center, IPAC, California Institute of Technology, Pasadena, CA 91125, USA

9 Spitzer Science Center, California Institute of Technology, MC 314-6, Pasadena, CA 91125, USA

10 Leiden Observatory, Leiden University, PO Box 9513, 2300 RA Leiden, The Netherlands

11 Department of Physics and Astronomy, University of Toledo, 2801 West Bancroft Street, Toledo, OH 43606, USA
12 Department of Physics \& Astronomy, University of Wyoming, Laramie, WY 82071, USA

13 Department of Astrophysical Sciences, Princeton University, Princeton, NJ 08544, USA

14 Max-Planck-Institut für Astronomie, Königstuhl 17, 69117 Heidelberg, Germany

15 Departamento de Astrofisica, Facultad de Ciencias Fisicas, Universidad Complutense Madrid, Ciudad Universitaria, Madrid, 28040, Spain

16 Tianjin Astrophysics Center, Tianjin Normal University, Tianjin 300387, PR China

17 Department of Physics and Astronomy, SUNY Stony Brook, Stony Brook, NY 11794-3800, USA

18 National Radio Astronomy Observatory, 520 Edgemont Road, Charlottesville, VA 22903, USA

19 Department of Physics \& Astronomy, McMaster University, Hamilton, Ontario L8S 4M1, Canada

${ }^{20} \mathrm{CEA} / \mathrm{DSM} / \mathrm{Irfu} /$ Service d'Astrophysique, UMR AIM, CE Saclay, 91191 Gif-sur-Yvette Cedex, France

${ }^{21}$ ICRAR, M468, University of Western Australia, 35 Stirling Hwy, Crawley, WA, 6009, Australia 